\title{
Acne fulminans in a Vietnamese boy successfully treated with prednisolone and ibuprofen: A case report
}

\author{
Quang Van Vu* (1)
}

Department of Pediatrics, Haiphong University of Medicine and Pharmacy, 72 A Nguyen Binh Khiem, Ngo Quyen, Haiphong, Vietnam

\section{Correspondence}

Quang Van Vu, Department of Pediatrics, Haiphong University of Medicine and Pharmacy, 72 A Nguyen Binh Khiem, Ngo Quyen, Haiphong, Vietnam

Email: vvquang@hpmu.edu.vn History

- Received: 14 August 2018

- Accepted: 14 September 2018

- Published: 30 September 2018

DOI :

https://doi.org/10.15419/bmrat.v5i9.483

\section{Check for updates}

\section{Copyright}

(c) Biomedpress. This is an openaccess article distributed under the terms of the Creative Commons Attribution 4.0 International license.

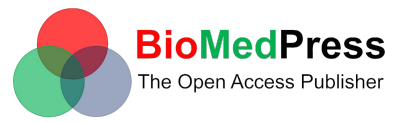

\begin{abstract}
Background: Acne fulminans (AF) is a rare disorder, the most severe form of the entire clinical spectrum of acne. It is characterized by the emergence of painful inflammatory nodules that turn into ulcers, concomitant with systemic manifestations. Here, we report for the first time a Vietnamese boy with Acne fulminans successfully treated with prednisolone and ibuprofen. Case presentation: The patient is a 14-year-old male teenager with moderate acne since the age of 13 . After a day of swimming, his acne suddenly became inflamed and painful lesions were present on his face and back. He had high fever and polyarthritis, and tests showed leukocytosis, neutrophilia, and elevated C-reactive protein (CRP). Severe infection was diagnosed and treated by addition of antibacterial therapy for a long period, however, the condition did not improve. When called upon to evaluate the patient, we established the diagnosis of acne fulminans and initiated treatment with prednisone (1 mg/kg/day) and ibuprofen (500 mg for two times daily). Following this treatment regimen, there was a rapid improvement. Conclusion: Herein, we have reported a Vietnamese boy with typical $A F$, but delayed diagnosis and treatment because of a rare condition. Our results support the role and benefit of prednisolone for AF treatment.

Key words: Acne fulminans, Acne maligna, Acne vulgaris, Acne with polyarthritis, Acute febrile ulcerative conglobate, Prednisolone
\end{abstract}

\section{INTRODUCTION}

Acne fulminans (AF) is a rare condition and the most severe form of acne. It is characterized by the sudden onset of painful and ulcerative pustules. The systemic symptoms include high fever and polyarthralgia $^{1,2}$. AF mainly affects young male adolescents with a history of acne vulgaris ${ }^{3,4}$. Laboratory findings typically show blood count abnormalities, with leukocytosis and neutrophilia, as well as elevated erythrocyte sedimentation rate and elevated C-reactive protein (CRP). The acne lesions are initially sterile; cultures from early lesions and blood are usually negative $^{5}$.

Treatment of AF can be difficult. There have been many reports of different treatments, including use of oral glucocorticoids and oral isotretinoin ${ }^{6}$. In the study herein, we report a case of this rare disease. To the best of our knowledge, this is the first report of AF in Vietnam.

\section{CASE PRESENTATION}

A 14-year-old boy was hospitalized at Hai Phong Children's Hospital due to a 4-week history of painful facial swelling, muscle and joint pain, and high fever $\left(39^{\circ} \mathrm{C}\right)$. He had acne vulgaris for the past 1 year. A month before the patient was referred to the hospital, he had gone for a swim and immediately after, facial swelling appeared. He was treated for 10 days in a district hospital with a combination of cefotaxime and gentamicin, and for 15 days in a dermatology hospital, but his clinical signs were not improved. He was otherwise healthy and had no remarkable family history.

The patient was diagnosed with diffuse inflammatory facial swelling and sepsis, and was treated with a combination of three antibiotics: rocephin, vancomycin, and metronidazole. However, after 15 days of treatment, the patient's clinical signs were not improved. He still had fluctuating fever, joint pain, facial swelling with yellow fluids (despite his daily care), and ulceronecrosis on the back (Figure 1).

Considering his medical history, current clinical signs, laboratory findings, as well as review of the literature, the patient was diagnosed with AF, a severe acne form. Antibiotics were rapidly stopped. $\mathrm{He}$ was treated with ibuprofen $(500 \mathrm{mg}$ ) for two times daily for 10 days, and prednisolone $(1 \mathrm{mg} / \mathrm{kg})$ for 24 hours. The cutaneous lesions were improved and the patient was able to walk normally in 7 days. $\mathrm{He}$ was discharged for outpatient treatment. Two weeks later, skin lesions healed completely with scarring 

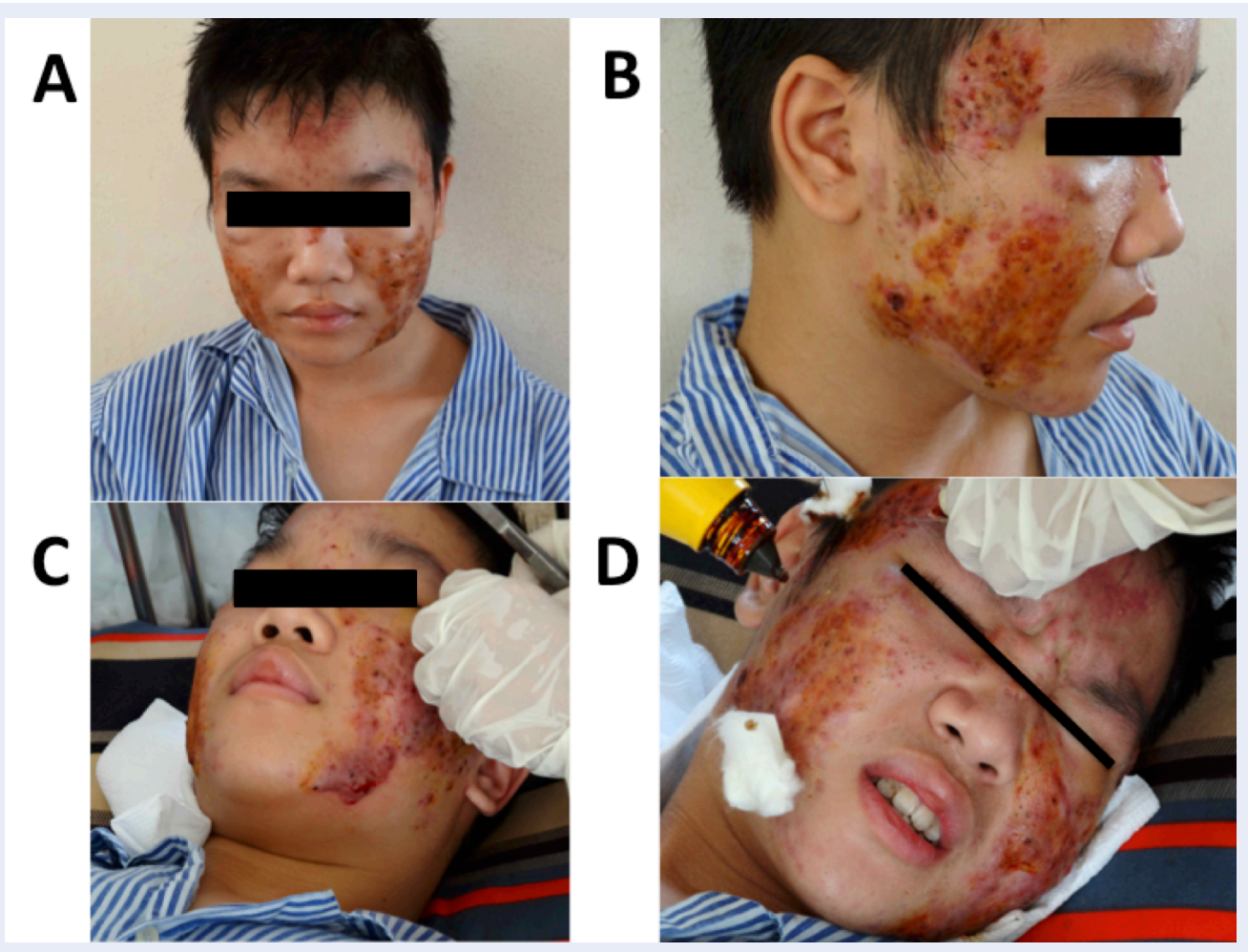

Figure 1: On the 40 th day in treatment with antibiotics. A, B: facial swelling; C, D: yellow fluids despite of daily care.

(Figure 2). The dose of prednisolone was progressively tapered to $0.5,0.25$, and $0.1 \mathrm{mg} / \mathrm{kg} / 24$ hours and stopped at the fifth month of treatment.

\section{DISCUSSION}

$\mathrm{AF}$ is a rare condition that is considered to be the most severe form of acne. The primary features of this disease include sudden onset, severe and often ulcerating acne, fever, polyarthritis, leukocytosis, and plaqueto$\mathrm{sis}^{2}$. The patient in this report showed a typical manifestation of AF. However, the diagnosis was delayed for at least 40 days. This issue can be explained by several reasons. First, AF is an extremely rare disease; indeed, its clinical manifestations resemble infectious diseases, which are common among Vietnamese children $^{7,8}$.

The etiopathogenesis of AF is not yet fully understood. However, it is thought to be an immunologically induced, systemic disease in which the triggering antigen is believed to be from Propionibacterium acnes. This bacterium can start to behave as a superantigen, triggering an exaggerated and disorganized antibody response, resulting in the alarming inflammatory symptoms ${ }^{3,6}$. Some reports have shown that almost all AF patients are male adolescents, suggesting that high blood testosterone levels during puberty may play an important role in the pathogenesis of acne fulminans.

Another theory is that AFs could be associated with an autoimmune etiology and genetic factors ${ }^{3,4,6}$. Normally, blood culture tests of AF patients are negative. Staphylococcus epidermidis and Propionibacterium acnes are predominant in skin culture; Pseudomonas, Staphylococcus aureus and Streptococcus pyogenes are more rare ${ }^{3}$. Staphylococcus epidermidis was isolated from the surface of the patient's skin; however, a direct relationship between these agents and the cutaneous eruption is unclear ${ }^{9}$.

The diagnosis of AF is usually clinical because of its unique clinical features.

Differential diagnosis includes: acne vulgaris, acne conglobate, rosacea fulminans, and synovitis-acnepustulosis-hyperostosis-osteitis (SAPHO) syndrome. In contrast with acne fulminans, the systemic symptoms of sudden onset acne are not common in acne vulgaris. Acne conglobata rarely affects the face and has a more chronic course without a sudden onset. The clinical feature of severe rosacea fulminans is somewhat similar to that of AFs, but usually it is seen 

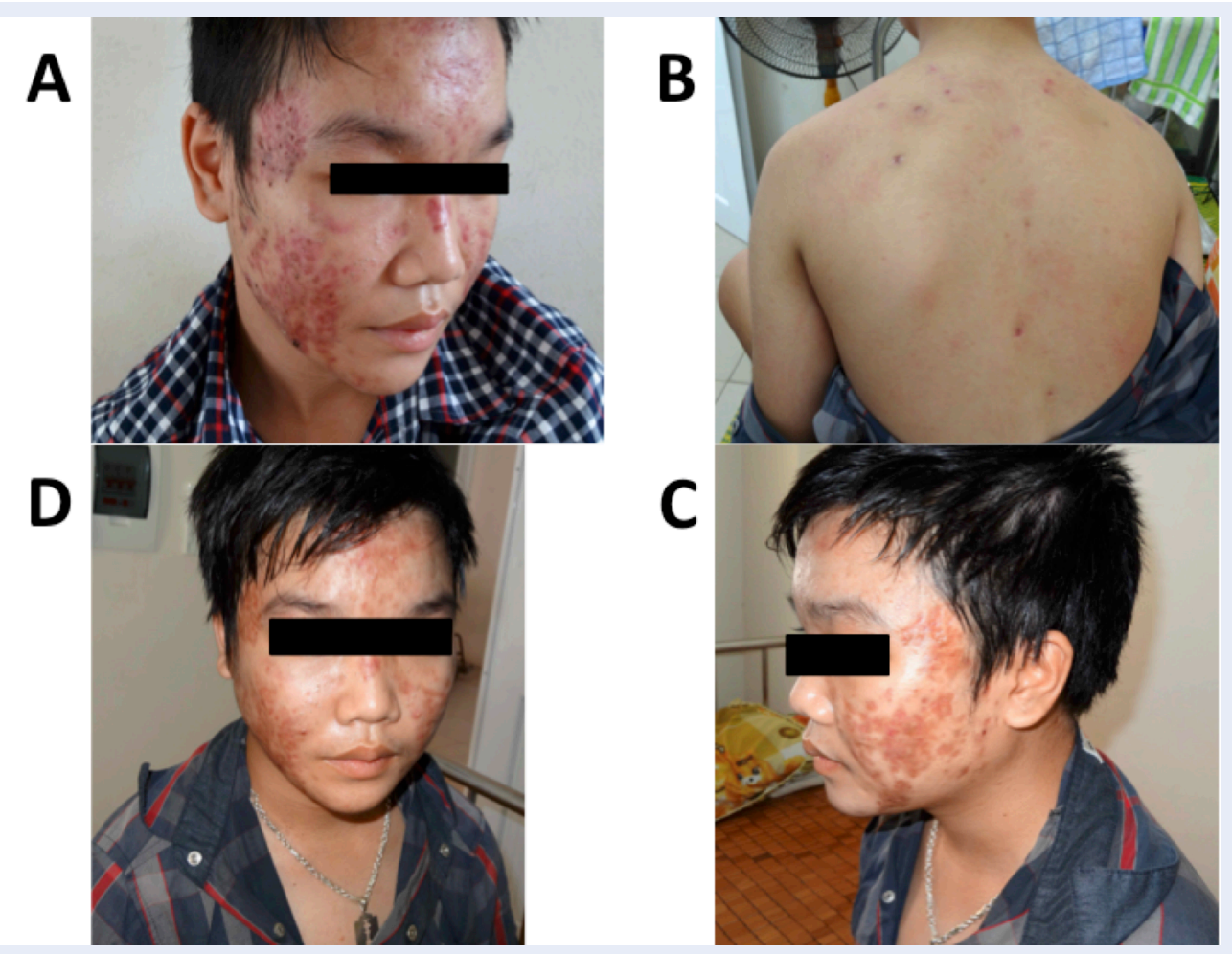

Figure 2: Acne fulminan. A: Patient after 7 days using prednisolone and ibuprofen; B,C,D: Patient after 21 days using prednisolone.

in women of higher age without a preceding history of acne ${ }^{1,6}$. The SAPHO syndrome is an association of distinct skin disorders with pustules with osteoarticular inflammation ${ }^{10}$; however, the patient in our study had no bony involvement nor impact on internal organs.

The treatment of AFs is still a challenge as there have been many reports of different treatments ${ }^{2,9,11}$. Our patient was treated with a combined use of antibiotics for a long period, although his clinical signs did not improve (Figure 1). According to some reports, AF does not respond to the conventional treatment for severe acne so the use of antibiotics alone is ineffective. However, antibiotics can be effective if there is microbial colonization ${ }^{1,6}$. Notably, along with general supportive care, systemic corticosteroids are the mainstay of the therapy ${ }^{5,6,11}$.

Our patient recovered significantly (Figure 2) after 10 days and 21 days of treatment with oral ibuprofen (500 mg, two times daily) and oral prednisolone ( $1 \mathrm{mg} / \mathrm{kg} / 24$ hours), respectively. The dose of prednisolone was progressively tapered and stopped at the $5^{\text {th }}$ month of treatment without relapse or adverse effects. Systemic glucocorticoids should be maintained at a higher dose for a period that varies from
2 to 4 months, and subsequently, should be gradually reduced to prevent relapse when the dosage is decreased $^{1,2,6}$

We did not give isotretinoin to the patient because this drug could trigger AF in some cases ${ }^{5,6}$. As reported in this case study, the patient some difficulty walking due to painful joints. However, after 7 days of treatment with ibuprofen and prednisolone, he was able to walk. The role of ibuprofen in AF treatment is unclear; this drug may help to decrease swelling, pain, or fever in patients with AF.

\section{CONCLUSIONS}

In summary, we describe a Vietnamese teenage boy with a typical phenotype of AF but delayed diagnosis. This is the first report of an AF case successfully treated by prednisone and ibuprofen.

\section{COMPETING INTERESTS}

The authors declare that they have no financial or other conflicts of interest.

\section{REFERENCES}

1. Tago $O$, Nagai $Y$, Matsushima $Y$, Ishikawa O. A case of acne fulminans successfully treated with cyclosporin A and prednisolone. Acta dermato-venereologica. 2011;91(3):337-338. Available from: Doi:10.2340/00015555-0796. 
2. Siadat AH, Bostakian A, Abtahi-Naeini B, Shahbazi M. Successful Treatment of Facial Acne Fulminans: Antimicrobial Agents and Oral Prednisolone as Promising Regimes. Case Reports in Dermatological Medicine. 2017;2017:7092910. Available from: Doi:10.1155/2017/7092910.

3. Pereira MF, Roncada EM, Oliveira CM, Monteiro R, Abreu MA Ortigosa LC. Acne fulminans and isotretinoin: case report. Anais Brasileiros de Dermatologia. 2011;86:983-5. Available from: Doi:10.1590/s0365-05962011000500017.

4. Zanelato TP, Gontijo GM, Alves CA, Pinto JC, Cunha PR. Disabling acne fulminans. Anais Brasileiros de Dermatologia. 2011;86:S9-12. Available from: Doi:10.1590/s036505962011000700001.

5. Proença NG. Acne fulminans. Anais Brasileiros de Dermatologia. 2017;92:8-10

6. Lages RB, Bona SH, Silva FV, Gomes AK, Campelo V. Acne fulminans successfully treated with prednisone and dapsone. Anais Brasileiros de Dermatologia. 2012;87:612-4. Available from: Doi:10.1590/s0365-05962012000400015.

7. Vu QV, Wada T, Le HT, Le HT, Nguyen ATV, Osamu O. Clinical and mutational features of Vietnamese children with X-linked agammaglobulinemia. BMC Pediatrics. 2014;14:129. Available from: Doi:10.1186/1471-2431-14-129.

8. Vu QV, Wada T, Tran TT, Ngo DN, Dinh TV, Nguyen CH. Severe congenital neutropenia caused by the ELANE gene mutation in a Vietnamese boy with misdiagnosis of tuberculosis and autoimmune neutropenia: a case report. BMC Hematology. 2015;15:2. Available from: DOI:10.1186/s12878-015-0020-x.

9. Giavedoni P, Mascaró-Galy JM, Aguilera P, Estrach-Panella T. Acne fulminans successfully treated with cyclosporine and isotretinoin. Journal of the American Academy of Dermatology. 2014;70:e38-e39.

10. Divya BL, Rao PN. SAPHO syndrome with acne fulminans and severe polyosteitis involving axial skeleton. Indian Dermatology Online Journal. 2016;7:414-7. Available from: Doi: 10.4103/2229-5178.190495.

11. Massa AF, Burmeister L, Bass D, Zouboulis CC. Acne Fulminans: Treatment Experience from 26 Patients. Dermatology (Basel Switzerland). 2017;233:136-40. Available from: Doi:10.1159/ 000473860 